\title{
Application of Multi-sensor Data Fusion Technology in Wheelbarrow Obstacle Avoidance System
}

\author{
Lina Duan ${ }^{1, a, *}$, Fei Tao ${ }^{2, b}$ \\ ${ }^{1}$ Department of Mechanical \&Electrical and Automation, Wuchang Shouyi University, Wuhan, \\ China \\ ${ }^{2}$ Department of Mechanical \&Electrical and Automation, Wuchang Shouyi University, Wuhan, \\ China \\ amiyuki2003@163.com, b1582024489@qq.com \\ *Corresponding author
}

Keywords: Wheelbarrow, Data Fusion, Avoidance System.

Abstract. This paper briefly introduces the development status of multi-sensor data fusion technology and knowledge of the basic principles of multi-sensor data fusion technology, and highlights based on BP Fuzzy Neural Network Information Fusion Method, and will change the method to the wheelbarrow obstacle avoidance system, set up given the physical model of the whole system for a wheelbarrow and wheelbarrow sensor information fusion systems. Debug and simulate in Matlab.

\section{Introduction}

In this paper, multi-sensor data fusion technology is studied as a focus. From the perspective of wheelbarrow avoidance angle related applications, the theory and practice of multi-sensor data fusion technology in the application of wheelbarrow obstacle avoidance system are carried out.

\section{Multi-sensor data fusion technology}

Multi-sensor information fusion is a comprehensive analysis and processing of unknown multi-source information, the key of the fusion method is to study the algorithm of information fusion. Multi-sensor information fusion technology has a wide range of applications and the file it involves is extremely rich.

Nowadays, the application of multi-sensor information fusion in the field of mobile robots has made great progress, the rich selections of the sensors and the increased accuracy of the sensors make the advantages of Multi - sensor data fusion technology keep increasing. As robot use the field is constantly increasing. The mobile robot that we expect not only to be able to complete a single function, but also can travel at the same time to feel only the surrounding environment changes, self-obstruction and navigation. Multi-sensor data fusion technology has been widely used in robot obstacle avoidance systems at present. 


\section{The Data Fusion Based on Fuzzy Neural Network}

\subsection{First Section Fuzzy Control}

Fuzzy control system is based on fuzzy mathematics, fuzzy language and fuzzy reasoning and is the use of computer control technology constitutes a closed-loop structure of the control system. The basic structure of the fuzzy controller and fuzzy controller of the fuzzy control system is shown in Figure 1.



Figure 1 Fuzzy controller structure diagram

As can be seen from Figure 1, the structure of the fuzzy controller consists of four parts: fuzzy input interface, fuzzy decision, knowledge base and clear output interface. The knowledge base consists of fuzzy "If-Then" rule base and database two parts. The fuzzy rules in the fuzzy rule base include expert knowledge or expert experience in related fields. Fuzzy database is used to define membership function, fuzzy series and scale transformation factor. Fuzzy decision-making is based on fuzzy rules and the requirements of the facts to reason, get the appropriate output. The fuzzy controller is set up with four steps: first select the appropriate input and output variables; then the fuzzy subset of the selected variable is defined; secondly through the fuzzy rules to establish the connection between input and output; finally, the output of fuzzy decision and defuzzification are carried out.

The focus of fuzzy controller is fuzzy inference, fuzzy reasoning process is to imitate the human brain reasoning method. The process of reasoning is based on the fuzzy control rules in the knowledge base. The whole reasoning process is based on the original requirements of the system, through the knowledge base in the fuzzy control rules to infer another new proposition. That is, the fuzzy control rules in the knowledge base into a corresponding mapping.

The result of the fuzzy decision is a vague language, and the fuzzy output required by the actual system is an accurate numerical value because the system can only be an exact amount. The state variables of the fuzzy controller in the fuzzy reasoning must be changed from the fuzzy quantity to the precise quantity in order to get the output quantity required by the actual system. Such a process from vague to clear is called clarity, and the commonly used method of clarity has the moment method and the maximum membership function method.

\subsection{Neural Network Control}

Neuron and neural network structures: Neuron is the basic unit of neural network, it is a multi-input single-output nonlinear device. 


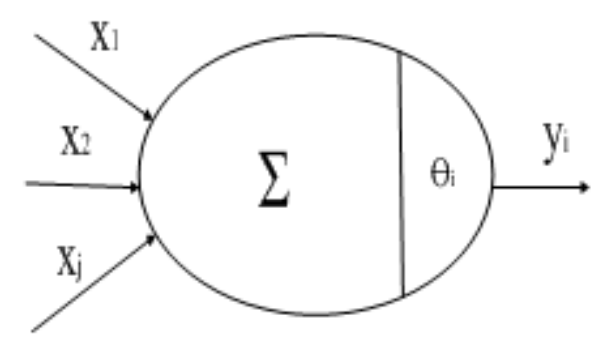

Figure 2 Basic structure of neurons

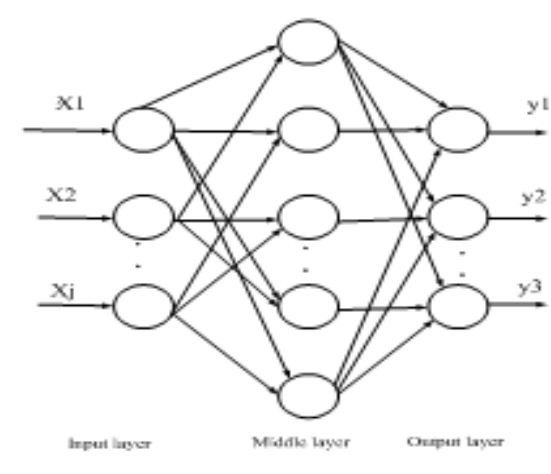

Figure 3 neural network diagrams

The basic structure of neurons is shown in Figure 2. Where $\mathrm{Xi}$ is the input state signal, $\theta \mathrm{i}$ is called the threshold, $\omega \mathrm{ij}$ is the weight, $\mathrm{Yi}$ is the system output signal, $\mu \mathrm{i}$ is the linear combination of the input signal, and $\varphi$ is the function of the unit.

The neuronal model is expressed as:

$$
\mu_{i}=\sum^{j} \omega_{i j} X_{j} \quad V i=\mu i-\theta i \quad y_{i}=\phi\left(V_{i}\right)
$$

Neural $=$ hetwork structure is usually used in the multi-layer structure of neurons, the classic neural network structure shown in Figure 3:

Neural network has a three-tier structure, in which the input layer and the middle layer is called hidden layer, the other is the network output layer. The network has input nodes, output nodes, and hidden nodes. The input data received by the network will pass through the information processing of the input layer and the middle layer (ie, the hidden layer), and then the information will be transmitted to the output node of the output layer through the information processing of each layer, and the appropriate result will be obtained.

The number of nodes in the input layer is determined by the actual input variable of the system. The nodes of the input layer are not only used to receive the input signal of the system, but also to buffer and save the signal. The number of nodes in the output layer also depends on the type of output signal. The number of nodes in the middle layer is related to the actual system and input and output. If the number of nodes is too large, the learning and training time will increase. However, if the node is too small, the fault tolerance of the system will be reduced, and the recognition ability will be weakened. The number must be considered in a number of factors. The transfer function generally selects a pure linear function or a Sigmoid type function in the neural network.

\section{Application of fuzzy neural network control in wheel protection system of wheelbarrow}

\subsection{Obstacle avoidance system}

Ultrasonic sensor based on TOF (time of flight) principle, TOF principle is the distance $=$ speed $\times$ time, such as the transfer speed of the sound waves is $0.3 \mathrm{~m} / \mathrm{ms}$ in a medium, if the distance is $10 \mathrm{~m}$, it takes $30 \mathrm{~ms}$ to reach. Then the distance is determined by calculating the time difference returned.

The ultrasonic sensor first generates a set of pulse signals, followed by the start timing. The system will set a return signal threshold to accept the sound return signal, the threshold will increase with time to reduce the sound signal to prevent the launch of the signal was received, the threshold of the return signal is set very high This will cause the ultrasonic sensor to have a blind spot in the blind area where the obstruction can't be detected. In addition there are several shortcomings of ultrasound, such as low scanning frequency, will produce cross-induction, especially when using the 
ultrasonic array, there will be echo attenuation, refraction and other issues. However, for the wheelbarrow system, the ultrasound is currently the most appropriate and most effective sensor.

\subsection{Obstacle avoidance system workflow}

Multi-channel ultrasonic sensor system work flow is as follows: the beginning of the NE555 issued $40 \mathrm{~K}$ square wave signal, change the signal through the pulse modulation signal after the drive sensor to send ultrasonic signals, and then select the switch control by multiple select the appropriate channel. In a certain delay after the opening of the corresponding return channel, when the receiver receives an ultrasonic sensor received by the obstruction of the signal back, the microcontroller generates an interrupt. Enter the interrupt service routine, the timer to suspend work, get the propagation time $t$, and then according to the TOF principle can get the distance of obstacles. The total flow chart of the obstacle avoidance system is shown in Figure 4:



Figure 4 Obstacle avoidance system total flow

\subsection{Application of fuzzy neural network in wheel protection system of wheellbarrow}

The obstacle avoidance behavior of the wheelbarrow in the process of traveling is as follows:

(1) Sensor obstacle avoidance system will carry out an obstacle detection after each period of time, it gets information about the obstacle based on the sensor and determines that the wheelbarrow is followed by a slowdown or steering action

(2) If the ultrasonic sensor has detected the information of the obstacle, the measured distance value of the ultrasonic sensor is used as the detection value of the obstacle in the direction; if the ultrasonic sensor does not detect the existence of the single obstacle information, the wheelbarrow keeps the original posture to continue driving.

(3) It receives and synthesizes the obstacle distance information obtained by the ultrasonic sensor and the electronic compass to obtain the target orientation information and is fed into a fuzzy neural network controller for data fusion

(4) It is the obstacle information to make the corresponding obstacle avoidance decision-making, to achieve to avoid obstacles

\subsubsection{Establishment of fuzzy neural network controller}

It uses fuzzy neural network data fusion algorithm and integrates the information detected by the 
multi-sensor to achieve the ultimate control of wheelbarrows at last. Fuzzy Neural Network Control wheelbarrow based obstacle avoidance controller is shown in Figure 5.

The controller has four inputs and two outputs in figure 5.Three of the inputs are the distance from the left, front and right obstructions, and the fourth input is the azimuth of the current position of the wheelbarrow, which can be measured by the electronic compass. The two outputs are the wheelbarrow turn left and turn right. When the wheelbarrow is in the process of traveling, the movement trajectory is adjusted according to the environmental information received by the sensor and the movement state of the vehicle body itself. After the obstacle is avoided, the target position information can be continued until the target position is reached.

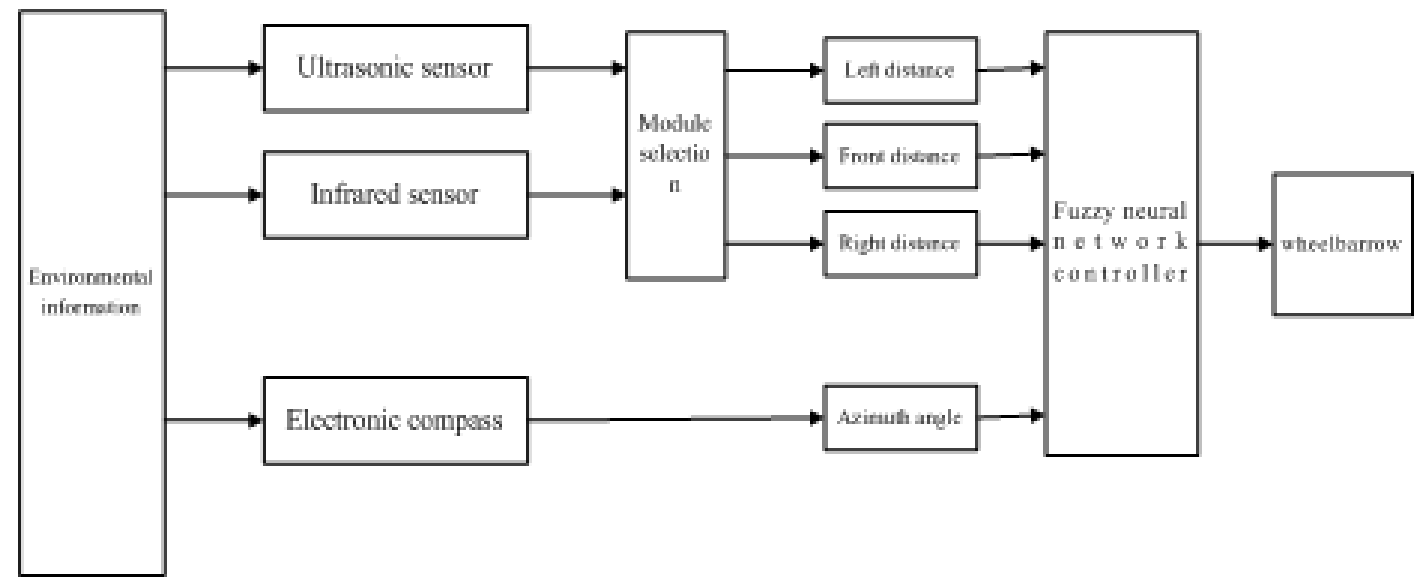

Figure 5 Fuzzy neural network controller block diagram

\subsubsection{The establishment of fuzzy rules}

According to the different environmental information, the travel path of the wheelbarrow is different. The fuzzy language of the fuzzy control rule is described as follows:

if left is far and front is far and right is far and head is $\mathrm{n}$ then go ahead;

if left is near and front is far and right is far and head is $\mathrm{z}$ then turn right;

As mentioned above, there are 24 words in the fuzzy language description, where the fuzzy language is expressed as: the output is left, front, right, head for the wheelbarrow left, front, right obstacle distance and direction angle; output for the wheelbarrow straight, turn left and turn right, far on behalf of distance, near representative distance, head fuzzy language variables for the N, Z, P, respectively, said the left, front, right.

\section{System debugging and simulation}

\subsection{Realization of obstacle avoidance algorithm for fuzzy neural network in Matlab}

\subsubsection{Set membership function}

First enter editor interface of the fuzzy neural network controller inside, and then enter the instruction Fuzzy in the command window of Matlab. If you want to edit an input and output variable, double-click to change the input and output variables can enter the corresponding membership function editing interface. The editor can be used to set and modify the parameters of the membership function corresponding to each language variable in the fuzzy reasoning system, such as the shape, scope, size of the membership function, etc. The system provides membership function with triangular, trapezoidal, Gaussian, bell and so on.

The distance velocity is chosen as the gauss $2 \mathrm{mf}$ membership function and the azimuth angle is 
chosen as the trim membership function in this system. Figure6, Figure 7 and Figure 8 are the input and output membership function curves established in this system.

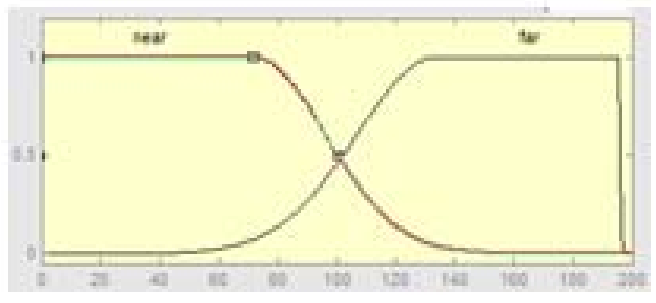

Figure 6 distance from each membership function

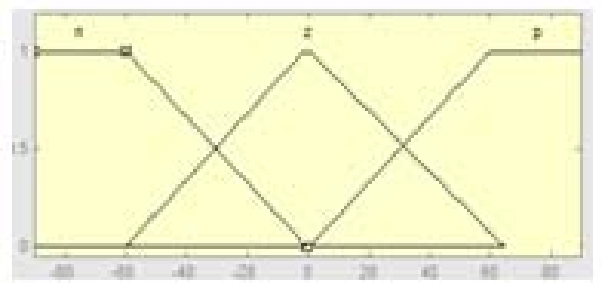

Figure 7 target angle membership function

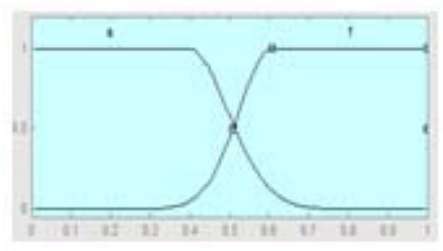

Figure 8 turning speed membership function

\subsubsection{Fuzzy control rules}

Find edit option in the fuzzy control interface and then click the Rules menu to display Rule Editor interface. Add a fuzzy control rule to the interface. The system involves 24 fuzzy control rules, fuzzy control rules generate interface shown in Figure9, can also generate the three-dimensional interface of the fuzzy control rules shown in Figure5at the same time.

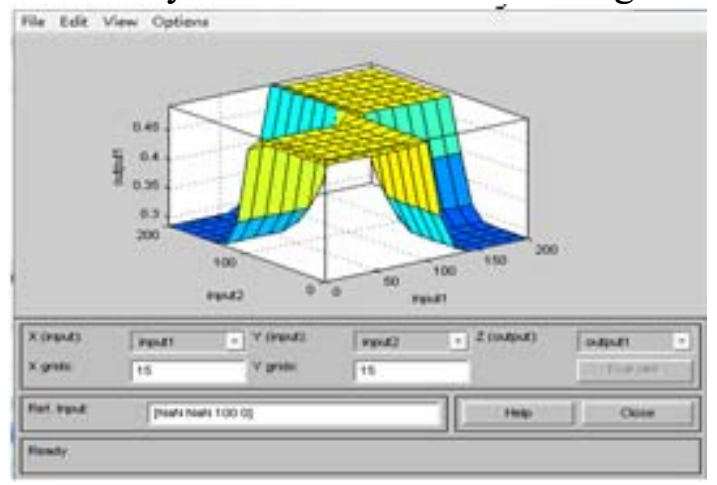

Figure 9 Fuzzy control rules generation interface 




Figure 10 Fuzzy control rules 3D interface

After all the above rules have been edited, generate a file named $*$.fis file. Since then it has completed the establishment of a fuzzy controller.

\subsubsection{Training of membership function}

Firstly, the training parameters of fuzzy neural network are constructed through the experience, and the membership function of fuzzy neural network controller is trained by parameter adjustment algorithm. Then the optimal membership function which can optimize the input variables is selected from the training samples. And take the test to calculate the neural network in the test sample on the error, once the error is no longer down, it can stop the training of the system.

\subsection{System simulation results}

We intend to be in the environment, in accordance with the fuzzy neural network integration of the guided wheelbarrow carried out a number of simulation avoidance training, so that the wheelbarrow can well avoid obstacles in the unknown environment, driving to the target location. It requires a wheelbarrow to start from the starting point and makes the wheelbarrow in a multi-obstacle simulation environment safe, collisionless to reach the end in the premise of meeting the safety requirements.Figure 11 is the obstacle avoidance trajectory in the Matlab simulation environment. It can be seen that the wheelbarrow has better obstacle avoidance performance during the running process.

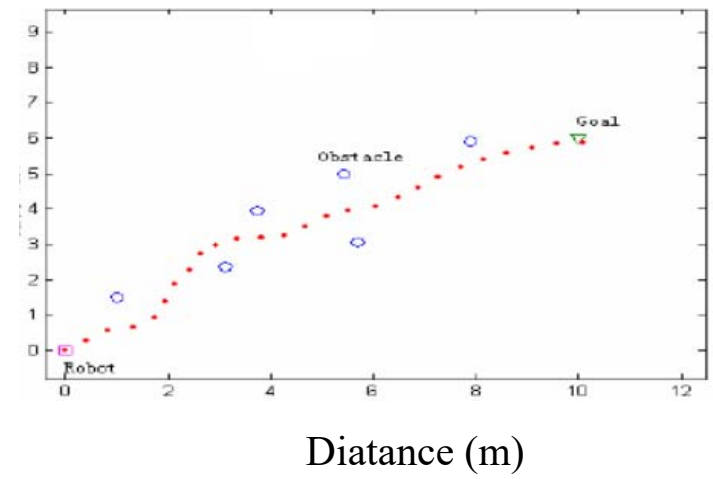

Figure 11 Fuzzy neural network simulation results

\section{Conclusions}

This paper discusses the algorithm of fuzzy neural network data fusion with wheelbarrow as the design object. This paper discusses the application of multi - sensor data fusion technology in wheelbarrow obstacle avoidance system. The main research contents of this paper include research 
on multi - sensor data fusion technology; the establishment of wheelbarrow obstacle avoidance system, selected for the various types of sensors; establishment of fuzzy neural network controller for system, design the Membership function and fuzzy rule base on this basis; using fuzzy logic toolbox in Matlab to complete the system of obstacle avoidance system.

\section{References}

[1] Li Qunfang. Single Chip Microcomputer and Interface Technology [M].Fourth edition. Beijing: Electronic Industry Press, 2009.

[2] Chen Boshi. Electric drag automatic control system. Beijing: Mechanical Industry Press,2003.

[3] Xue Dingyu, Chen Yangquan. System Simulation Technology and Application Based on MATLAB / Simulink [M].Beijing: Tsinghua University Press,2002.

[4] Chen Zhiwang.STM32 embedded microcontrollers quickly get started.Beijing:Electronic Industry Press,2014.

[5] Zhang Yi,Luo Yuan,Zheng Taixiong.Mobile Robot Technology and Its Application [M].Beijing: Electronic Industry Press, 2007.

[6] Yan Pingfan, Zhang Changshui. Artificial Neural Network and Simulated Evolutionary Computation. Second edition. Beijng:Tsinghua University Press,2005.

[7] Li Guoyong.Intelligent Control and Its Realization of [M].Beijing:Electronic Industry Press,2006.

[8] He You,Wang Guohong,Peng Yingning.Multi - sensor Information Fusion and Application [M].Second edition.Beijing:Electronic Industry Press,2007.

[9] Yang Wanhai.Multi - sensor Data Fusion and Its Application [M].Xi'an: Xi'an University of Electronic Science and Technology Press,2004. 